\title{
Closed-form Generalized Power Correlation Coefficient of the Hoyt Fading Signal
}

\author{
José Ricardo Mendes, Michel Daoud Yacoub, and Gustavo Fraidenraich
}

\begin{abstract}
Exact, closed-form, and general expressions of the marginal and joint moments as well as of the correlation coefficient of the instantaneous powers of two Hoyt (Nakagami-q) signals are derived. All provided statistics are expressed as finite sums of simple functions of the model parameters. The model allows for environments where the variances of the quadrature components of a signal are different from their counterparts of the other signal. Some numerical results illustrate the generalized power correlation coefficient provided in this work, simulations support the theoretical results, and an approximation to the envelope correlation coefficient of the Hoyt model is proposed.
\end{abstract}

Index Terms-Correlation coefficient, fading channel, Hoyt distribution, power statistics.

\section{INTRODUCTION}

$I^{\prime}$ $\mathrm{N}$ WIRELESS communications, the signal envelope fluctuates randomly throughout the propagation environment in a fast fading condition. Among the several distributions used to describe the signal envelope, the Rayleigh, Rice, Hoyt (Nakagami-q), and Nakagami-m constitute the most representative ones. In particular, the Hoyt model [1] considers the in-phase and quadrature signal components as Gaussians with zero means and arbitrary variances. For the case of identical variances, the Hoyt distribution reduces to the Rayleigh one.

In recent years, different statistics concerning the Hoyt model have been investigated [2]-[4]. However, to the best of the authors' knowledge, neither the envelope correlation coefficient nor the power correlation coefficient of two Hoyt signals has been reported in the literature. This work provides an exact, closed-form, and general expression for the power correlation coefficient. To this end, marginal and joint moments of arbitrary positive integer orders of the instantaneous powers are derived. All these statistics are obtained for both stationary ${ }^{1}$ and nonstationary environments.

It is widely known that, for the Rayleigh model [5] and for the Ricean model [6], the power correlation coefficient is a simple and accurate approximation to the envelope correlation coefficient, whose exact mathematical treatment is rather complicated. In fact, for the Rayleigh case, the well-known results of the coherence bandwidth and the coherence distance (or time) [5], two important parameters used as reference for the frequency separation and space separation in diversity systems, have been obtained from the power correlation coefficient

Manuscript received July 15, 2005. The associate editor coordinating the review of this letter and approving it for publication was Prof. Giorgio Taricco.

The authors are with the Dept. of Communications, School of Electrical and Computer Engineering, University of Campinas, Brazil (e-mail: \{ricardo, michel, gf\}@wisstek.org).

Digital Object Identifier 10.1109/LCOMM.2006.02009.

${ }^{1}$ In this work, the term stationary environment designates the environment where the variances of the quadrature components of one signal are equal to their counterpart of the other signal. instead of the envelope one. As will be seen in this work, that approximation is also efficient for the Hoyt model.

Section II introduces the Hoyt model. Section III presents the generalized power statistics. Section IV analyzes the theoretical results, compares them with simulation ones, and proposes approximations to the power correlation coefficient for non-integer orders. Section V concludes this work.

\section{Signal Model}

Consider two received signals, $S_{1}$ and $S_{2}$, and their respective envelopes, $R_{1}$ and $R_{2}$. The instantaneous power $W_{i}$ (or squared envelope $R_{i}^{2}$ ) of each signal is given by

$$
W_{i}=R_{i}^{2}=X_{i}^{2}+Y_{i}^{2} \quad i=1,2
$$

where $X_{i}$ and $Y_{i}$ are, respectively, the in-phase and quadrature components of $S_{i}$.

In the Hoyt model, the joint probability density function (JPDF) of $X_{1}, Y_{1}, X_{2}$, and $Y_{2}$ is

$$
\begin{array}{r}
f_{X_{1,2} Y_{1,2}}\left(x_{1}, y_{1}, x_{2}, y_{2}\right)=\frac{1}{4 \pi^{2} \sigma_{X_{1}} \sigma_{Y_{1}} \sigma_{X_{2}} \sigma_{Y_{2}}\left(1-\rho^{2}\right)} \\
\times \exp \left[-\frac{1}{2\left(1-\rho^{2}\right)}\left(\frac{x_{1}^{2}}{\sigma_{X_{1}}^{2}}+\frac{y_{1}^{2}}{\sigma_{Y_{1}}^{2}}+\frac{x_{2}^{2}}{\sigma_{X_{2}}^{2}}+\frac{y_{2}^{2}}{\sigma_{Y_{2}}^{2}}\right.\right. \\
-2 \mu_{1} \frac{x_{1} x_{2}}{\sigma_{X_{1}} \sigma_{X_{2}}}-2 \mu_{2} \frac{x_{1} y_{2}}{\sigma_{X_{1}} \sigma_{Y_{2}}} \\
\left.\left.+2 \mu_{2} \frac{x_{2} y_{1}}{\sigma_{X_{2}} \sigma_{Y_{1}}}-2 \mu_{1} \frac{y_{1} y_{2}}{\sigma_{Y_{1}} \sigma_{Y_{2}}}\right)\right]
\end{array}
$$

where $\sigma_{X_{i}}^{2}$ and $\sigma_{Y_{i}}^{2}$ are, respectively, the variances of $X_{i}$ and $Y_{i}, i=1,2, \mu_{1}=E\left\{X_{1} X_{2}\right\} /\left(\sigma_{X_{1}} \sigma_{X_{2}}\right)=$ $E\left\{Y_{1} Y_{2}\right\} /\left(\sigma_{Y_{1}} \sigma_{Y_{2}}\right), \mu_{2}=E\left\{X_{1} Y_{2}\right\} /\left(\sigma_{X_{1}} \sigma_{Y_{2}}\right)=$ $-E\left\{Y_{1} X_{2}\right\} /\left(\sigma_{Y_{1}} \sigma_{X_{2}}\right)$, and $\rho=\sqrt{\mu_{1}^{2}+\mu_{2}^{2}} \leq 1$. The coefficients $\mu_{1}$ and $\mu_{2}$ depend on the distance between the reception points, on the frequency difference between the transmitted signals, among others [5].

The present model is general and encompass as special cases: (i) stationary environments, for which $\sigma_{X_{1}}=\sigma_{X_{2}}$ and $\sigma_{Y_{1}}=\sigma_{Y_{2}}$; (ii) the Rayleigh distribution, for wich $\sigma_{X_{i}}=\sigma_{Y_{i}}$, $i=1,2$.

\section{Generalized Power Statistics}

In this section, the marginal moment, the joint moment, and the correlation coefficient of arbitrary positive integer orders of the instantaneous powers are derived. With the purpose of expressing these statistics in more compact forms, they will be presented in terms of the normalized instantaneous powers $\hat{W}_{i}$, which are given by

$$
\hat{W}_{i}=\frac{W_{i}}{E\left\{W_{i}\right\}}=\frac{X_{i}^{2}+Y_{i}^{2}}{\sigma_{X_{i}}^{2}+\sigma_{Y_{i}}^{2}} \quad i=1,2
$$




\section{A. Power Marginal Moment}

Using (1) and the independence of $X_{i}$ and $Y_{i}, i=1,2$, the moment $E\left\{W_{i}^{n}\right\}$ (n positive integer) can be calculated as

$$
E\left\{W_{i}^{n}\right\}=\sum_{m=0}^{n}\left[\left(\begin{array}{c}
n \\
m
\end{array}\right) E\left\{X_{i}^{2 m}\right\} E\left\{Y_{i}^{2 n-2 m}\right\}\right]
$$

The moment $E\left\{Z^{2 n}\right\}$ ( $n$ integer) of a Gaussian random variable $Z$ with zero mean and variance $\sigma_{Z}^{2}$ is well-known

$$
E\left\{Z^{2 n}\right\}=(2 n-1) ! ! \sigma_{Z}^{2 n}
$$

where $(-1) ! !=1$, and $(2 n-1) ! !=1 \cdot 3 \cdot \ldots \cdot(2 n-1)$ for $n \geq 1$. Using (3), (4), and (5)

$$
E\left\{\hat{W}_{i}^{n}\right\}=\frac{n !}{2^{n}\left(1+\eta_{i}\right)^{n}} \sum_{m=0}^{n}\left[\frac{(2 m) !(2 n-2 m) !}{[(m) !(n-m) !]^{2}} \eta_{i}^{m}\right]
$$

where $\eta_{i}=\sigma_{X_{i}}^{2} / \sigma_{Y_{i}}^{2}, i=1,2$. In particular, for the Rayleigh model, $\eta_{1}=\eta_{2}=1$.

\section{B. Power Joint Moment}

From (3), the joint moment of the normalized instantaneous powers can be expressed as

$$
\begin{aligned}
& E\left\{\hat{W}_{1}^{n_{1}} \hat{W}_{2}^{n_{2}}\right\}=\frac{1}{\left(1+\eta_{1}\right)^{n_{1}}\left(1+\eta_{2}\right)^{n_{2}}} \\
& \times \sum_{m_{1}=0}^{n_{1}} \sum_{m_{2}=0}^{n_{2}}\left[\left(\begin{array}{c}
n_{1} \\
m_{1}
\end{array}\right)\left(\begin{array}{c}
n_{2} \\
m_{2}
\end{array}\right) \eta_{1}^{m_{1}} \eta_{2}^{m_{2}} C_{G}\right]
\end{aligned}
$$

where $n_{1}$ and $n_{2}$ are positive integers, $\eta_{i}=\sigma_{X_{i}}^{2} / \sigma_{Y_{i}}^{2}, i=1,2$, and the coefficient $C_{G}$ is conveniently defined as

$$
C_{G} \triangleq \frac{E\left\{X_{1}^{2 m_{1}} Y_{1}^{2 n_{1}-2 m_{1}} X_{2}^{2 m_{2}} Y_{2}^{2 n_{2}-2 m_{2}}\right\}}{\sigma_{X_{1}}^{2 m_{1}} \sigma_{Y_{1}}^{2 n_{1}-2 m_{1}} \sigma_{X_{2}}^{2 m_{2}} \sigma_{Y_{2}}^{2 n_{2}-2 m_{2}}}
$$

Using (7b) and the definition of joint moment

$$
\begin{aligned}
C_{G} & =\int_{-\infty}^{\infty} \int_{-\infty}^{\infty} \int_{-\infty}^{\infty} \int_{-\infty}^{\infty} \frac{x_{1}^{2 m_{1}} y_{1}^{2 n_{1}-2 m_{1}} x_{2}^{2 m_{2}} y_{2}^{2 n_{2}-2 m_{2}}}{\sigma_{X_{1}}^{2 m_{1}} \sigma_{Y_{1}}^{2 n_{1}-2 m_{1}} \sigma_{X_{2}}^{2 m_{2}} \sigma_{Y_{2}}^{2 n_{2}-2 m_{2}}} \\
& \times f_{X_{1,2} Y_{1,2}}\left(x_{1}, y_{1}, x_{2}, y_{2}\right) d x_{1} d y_{1} d x_{2} d y_{2}
\end{aligned}
$$

The JPDF $f_{X_{1,2} Y_{1,2}}\left(x_{1}, y_{1}, x_{2}, y_{2}\right)$ is given in (2). After a tedious procedure of manipulation, the authors have found an exact and closed-form expression for $C_{G}$

$$
\begin{aligned}
C_{G} & =\left(2 m_{1}\right) !\left(2 n_{1}-2 m_{1}\right) ! \mu_{1}^{2 n_{1}-2 m_{1}} \mu_{2}^{2 m_{1}} \\
& \times \sum_{i_{1}=0}^{m_{1}} \sum_{j_{1}=0}^{n_{1}-m_{1}} \sum_{i_{2}=0}^{2 m_{1}-2 i_{1}} \sum_{j_{2}=\left\lceil i_{2} / 2\right\rceil}^{n_{1}+\left\lfloor i_{2} / 2\right\rfloor-m_{1}-j_{1}}\left[\frac{(-1)^{i_{2}}}{2^{i_{1}+j_{1}}}\right. \\
& \times \frac{\left[2\left(n_{1}+n_{2}-m_{2}-i_{1}-j_{1}-j_{2}\right)-1\right] ! !}{\left(2 m_{1}-2 i_{1}-i_{2}\right) !\left[2\left(n_{1}-m_{1}-j_{1}-j_{2}\right)+i_{2}\right] !} \\
& \left.\times \frac{\left(2 m_{2}+2 j_{2}-1\right) ! !}{i_{1} ! i_{2} ! j_{1} !\left(2 j_{2}-i_{2}\right) !}\left(\frac{\mu_{1}}{\mu_{2}}\right)^{2 i_{2}-2 j_{2}} \frac{\left(1-\rho^{2}\right)^{i_{1}+j_{1}}}{\mu_{1}^{2 j_{1}} \mu_{2}^{2 i_{1}}}\right]
\end{aligned}
$$

where $n_{1}, n_{2}, m_{1}$, and $m_{2}$ are non-negative integers satisfying $m_{i} \leq n_{i}, i=1,2,\lfloor\nu\rfloor$ is the greatest integer less than or equal to $\nu$, and $\lceil\nu\rceil$ is the smallest integer greater than or equal to $\nu$. From (9), $C_{G}$ is independent of $\sigma_{X_{1}}, \sigma_{Y_{1}}, \sigma_{X_{2}}$, and $\sigma_{Y_{2}}$.

\section{Power Correlation Coefficient}

By definition, the correlation coefficient of $\hat{W}_{1}^{n_{1}}$ and $\hat{W}_{2}^{n_{2}}$ ( $n_{1}$ and $n_{2}$ positive integers) is

$$
\delta_{n_{1}, n_{2}}=\frac{E\left\{\hat{W}_{1}^{n_{1}} \hat{W}_{2}^{n_{2}}\right\}-E\left\{\hat{W}_{1}^{n_{1}}\right\} E\left\{\hat{W}_{2}^{n_{2}}\right\}}{\sqrt{\operatorname{Var}\left\{\hat{W}_{1}^{n_{1}}\right\} \operatorname{Var}\left\{\hat{W}_{2}^{n_{2}}\right\}}}
$$

where

$$
\operatorname{Var}\left\{\hat{W}_{i}^{n_{i}}\right\}=E\left\{\hat{W}_{i}^{2 n_{i}}\right\}-E^{2}\left\{\hat{W}_{i}^{n_{i}}\right\} \quad i=1,2
$$

In (10), the joint moment $E\left\{\hat{W}_{1}^{n_{1}} \hat{W}_{2}^{n_{2}}\right\}$ is found through (7a) and (9), whereas the marginal moments are obtained directly from (6). Since $n_{1}$ and $n_{2}$ are arbitrary positive integers, the power correlation coefficient provided here is rather general. For the particular case in which $n_{1}=n_{2}=1$

$$
\delta_{1,1}=\frac{\mu_{1}^{2}\left(1+\eta_{1} \eta_{2}\right)+\mu_{2}^{2}\left(\eta_{1}+\eta_{2}\right)}{\sqrt{\left(1+\eta_{1}^{2}\right)\left(1+\eta_{2}^{2}\right)}}
$$

It is important to mention that the statistical behavior of the Hoyt signal power is symmetrical for $0 \leq \eta_{i} \leq 1$ and $1 \leq \eta_{i} \leq \infty$. Thus, the range $0 \leq \eta_{i} \leq 1$ describes completely the influence of $\eta_{i}$ on the power statistics. Moreover, from (3) and (10), it is readily seen that the correlation coefficient of $W_{1}^{n_{1}}$ and $W_{2}^{n_{2}}$ is equal to the correlation coefficient of $\hat{W}_{1}^{n_{1}}$ and $\hat{W}_{2}^{n_{2}}$. Therefore, $\delta_{n_{1}, n_{2}}$ is also the correlation coefficient of $W_{1}^{n_{1}}$ and $W_{2}^{n_{1}}$.

\section{Numerical Results and Simulations}

In this section, we investigate, in both space domain and frequency domain, the power correlation coefficient of the Hoyt model for different values of $n_{1}=n_{2}=n$ and $\eta_{1}=\eta_{2}=\eta$ (stationary environments). In order to verify the correctness of the provided statistics, all of the theoretical curves are contrasted with simulation results. We also propose approximations to the correlation coefficient for non-integer orders of the instantaneous powers.

As for the parameters $\mu_{1}$ and $\mu_{2}$, we shall assume the physical model described by Jakes, in which [5]

$$
\mu_{1}=\frac{J_{0}(\beta d)}{1+(\Delta \omega \bar{T})^{2}} \quad \mu_{2}=-\frac{\Delta \omega \bar{T} J_{0}(\beta d)}{1+(\Delta \omega \bar{T})^{2}}
$$

where $\beta$ is the phase constant, $d$ is the distance between the reception points, and $\Delta \omega$ is the frequency difference between the transmitted signals. For a mobile receiver, $d=v \tau$, where $v$ is the mobile velocity, and $\tau$ is the time.

Throughout the following analysis, for clarity, we denote

- $\delta_{n, n}(d), \delta_{n, n}(\tau), \delta_{n, n}(\Delta \omega)$ : space, time, frequency correlation coefficient of the instantaneous powers;

- $d_{c}, \tau_{c}, \Delta \omega_{c}$ (coherence distance, time, bandwidth): space, time, frequency separation above which the envelope correlation coefficient is below a certain value.

\section{A. Influence of $n$}

Fig. 1 and Fig. 2 illustrate some theoretical curves (solid lines) and simulation results (dotted points) of $\delta_{n, n}(d)$ and $\delta_{n, n}(\Delta \omega)$ for $\eta=0.5$ and different values of $n$. It can be noted that: (i) the smaller the $n$ the stronger the correlation coefficients; (ii) when $n \rightarrow \infty$, both $\delta_{n, n}(d)$ and $\delta_{n, n}(\Delta \omega)$ 


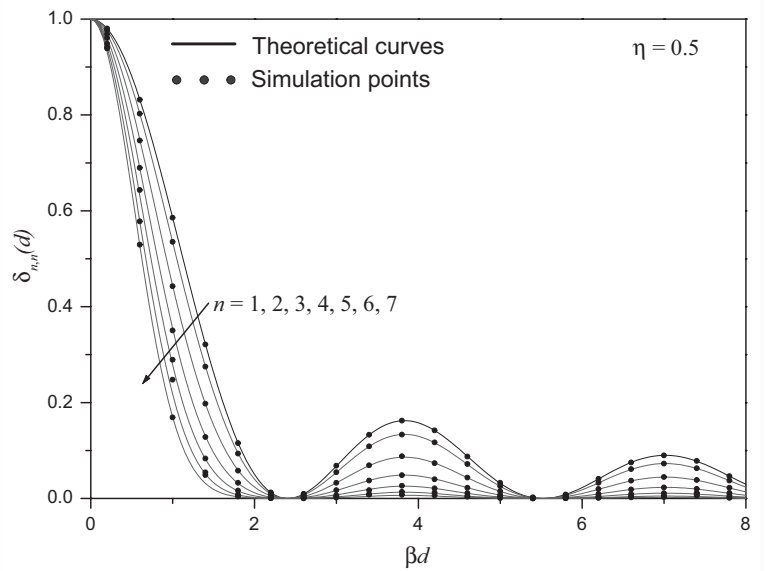

Fig. 1. Space correlation coefficient $(\Delta \omega=0)$ for $\eta_{1}=\eta_{2}=0.5$ and some values of $n_{1}=n_{2}=n$.

tend towards zero but at the origin; (iii) the theoretical and simulation results match excellently.

\section{B. Non-integer Orders of the Instantaneous Powers}

1) Non-integers $\nu>1$ : From Fig. 1 and Fig. $2, \delta_{n, n}$ is close to $\delta_{n+1, n+1}$. Thus, for a positive non-integer $\nu$ satisfying $n<\nu<n+1$ ( $n \geq 1$ integer), the correlation coefficient of $W_{1}^{\nu}$ and $W_{2}^{\nu}$, namely $\delta_{\nu, \nu}$, can be well-approximated by the linear interpolation $\delta_{\nu, \nu} \doteq(\nu-n)\left(\delta_{n+1, n+1}-\delta_{n, n}\right)+\delta_{n, n}$.

2) Envelope Correlation Coefficient $(\nu=0.5)$ : For the Rayleigh model, which is a particular case of the Hoyt model, the envelope correlation coefficient $\left(\delta_{0.5,0.5}\right)$ is well approximated by the power correlation coefficient $\left(\delta_{1,1}\right)$ [5]. Based on this and on the proximity of two subsequent curves of $\delta_{n, n}$ in Fig. 1 and in Fig. 2 (in particular, on $\delta_{1,1} \doteq \delta_{2,2}$ ), we conclude that the approximation $\delta_{0.5,0.5} \doteq \delta_{1,1}$ is also efficient for the Hoyt model.

\section{Influence of $\eta$}

Interestingly, for stationary environments $\left(\eta_{1}=\eta_{2}=\eta\right)$, $\mu_{1}$ and $\mu_{2}$ as provided in (12) produce a $\delta_{1,1}(d)$ (as well as $d_{c}$ and $\tau_{c}$ ) independent of $\eta$ : from (12) and $\Delta \omega=0, \mu_{2}=0$; hence, substituting $\mu_{2}=0$ and $\eta_{1}=\eta_{2}$ into (11), $\delta_{1,1}(d)=\mu_{1}^{2}$ (independent of $\eta$ ).

Fig. 3 shows theoretical (solid lines) and simulation (dotted points) results of $\delta_{1,1}(\Delta \omega)$ for different values of $\eta$. Note that, since the Hoyt model is symmetrical for $0 \leq \eta \leq 1$ and $1 \leq \eta \leq \infty$, the frequency correlation coefficient and the coherence bandwidth $\Delta \omega_{c}$ are maximal when $\eta=1$ (Rayleigh model). Again, an excellent agreement between the theoretical and simulation results is observed.

\section{CONCLUSion}

This work has provided exact, closed-form, and general expressions of the marginal and joint moments as well as of the correlation coefficient of the instantaneous powers of two Hoyt signals. All statistics have been expressed as finite sums of simple functions of the model parameters. Simple

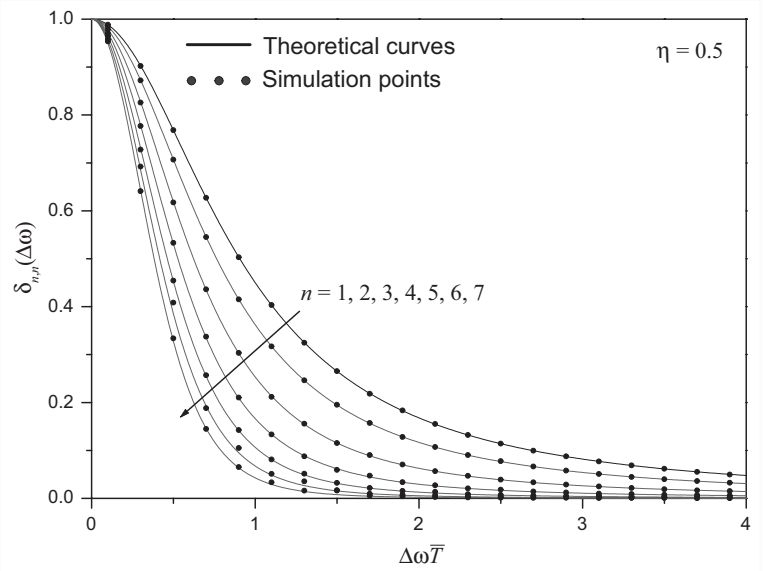

Fig. 2. Frequency correlation coefficient $(d=0)$ for $\eta_{1}=\eta_{2}=0.5$ and some values of $n_{1}=n_{2}=n$.

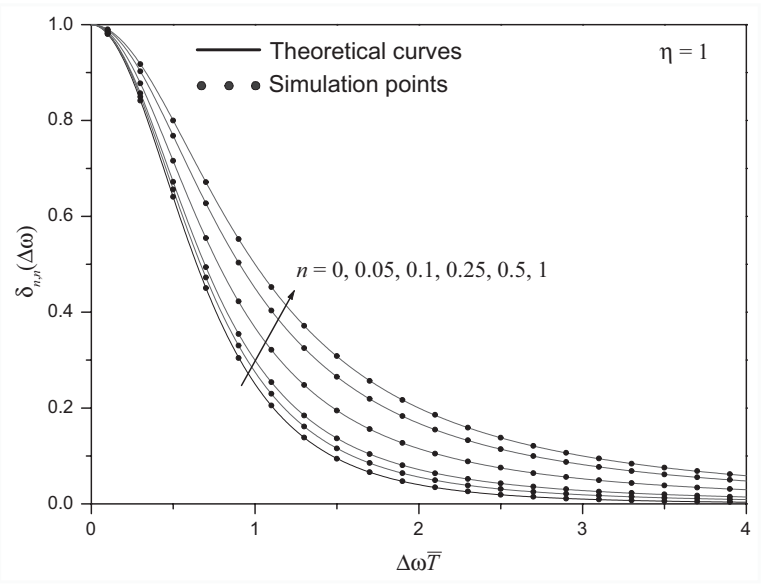

Fig. 3. Frequency correlation coefficient $(d=0)$ for $n=1$ and some values of $\eta_{1}=\eta_{2}=\eta$.

and accurate approximations to non-integer orders of the instantaneous powers have been proposed. Theoretical results have been supported by contrasting with simulation ones.

\section{REFERENCES}

[1] R. S. Hoyt, "Probability Functions for the Modulus and Angle of the Normal Complex Variate," Bell Syst. Tech. J., vol. 26, pp. 318-359, Apr. 1947.

[2] N. Youssef, W. Elbahri, M. Pätzold, and S. Elasmi, "On the Crossing Statistics of Phase Processes and Random FM Noise in Nakagami-q Mobile Fading Channels," IEEE Trans. Wireless Commun., vol. 4, pp. 24-29, Jan. 2005.

[3] J. Cheng and T. Berger, "Capacity of Nakagami-q (Hoyt) fading channels with channel side information," in Proc. International Conference on Communication Technology, vol. 2, Apr. 2003, pp. 1915-1918.

[4] C.-D. Iskander and P. T. Mathiopoulos, "Exact Performance Analysis of Dual-Branch Coherent Equal-Gain Combining in Nakagami-m, Rice and Hoyt Fading," in Proc. IEEE Southeast Conference, no. 8-10, Apr. 2005, pp. 233-239.

[5] W. C. Jakes, Microwave Mobile Communications. New York: Willey, 1997.

[6] Y. Karasawa and H. Iwai, "Modeling of signal envelope correlation of line-of-sight fading with applications to frequecy correlation analysis," IEEE Trans. Commun., vol. 42, 1994. 